\title{
PERFORMANCE IMPROVEMENT OF CZT DETECTORS BY LINE ELECTRODE GEOMETRY
}

\author{
LAN ZHANG, WEI ZHANG, JUN LI, YANQING LIU \\ Nuctech Company Limited \\ Beijing, 100084, China \\ zhanglan@nuctech.com \\ YULAN LI, YUANJING LI, YINONG LIU, JIANQIANG FU, HAO JIANG, ZHI DENG, \\ TAO XUE, JIE LUO \\ Tsinghua University \\ Beijing, 100084, China \\ yulanli@mail.tsinghua.edu.cn \\ Published 25 February 2014
}

\begin{abstract}
Due to their high efficiency and high energy resolution, applications of CdZnTe (CZT) detectors have spread into many areas such as room temperature detectors. To improve the performance and decrease the effects of hole trapping and crystal non-uniformity, special contact geometries are applied to the detectors, such as Coplanar, 3D pixel, Frisch grid, Capture and so on. In this paper, we introduce a new line electrode geometry to greatly improve CZT detector performance. A line anode collects electrons, while a planar cathode collects holes. Due to the low electric field close to the cathode and the low hole $\mu \tau$ value, this geometry is also a single charge sensitive structure. The calculated energy resolution of a $20 \mathrm{~mm}$ x $20 \mathrm{~mm}$ x $15 \mathrm{~mm}$ detector could be improved up to $1.51 \%$ for $662 \mathrm{keV}$ gamma rays. Both simulations and experimental results are presented here.
\end{abstract}

Keywords: CdZnTe; CdTe; semiconductor detectors.

\section{Introduction}

CdZnTe (CZT) detectors are widely applied in different fields. Spectroscopy and photon counting imaging are two main applications. Here we discuss the CZT detectors used for gamma and X-ray detection. As opposed to thin CdZnTe pixel detectors used for X-ray imaging at energies lower than $300 \mathrm{keV}$, a much thicker CZT crystal should be used for high energy gamma ray detection. However, crystal defects and charge trapping greatly decrease the detector performance. In order to obtain high energy resolution together with high detection efficiency, several contact geometries have been applied, such as Coplanar, 3D pixel, Frisch grid, Capture and so on. ${ }^{1-4}$ These geometries improve the CZT performance mainly due to a higher single polarity charge sensitivity.

This is an Open Access article published by World Scientific Publishing Company. It is distributed under the terms of the Creative Commons Attribution 3.0 (CC-BY) License. Further distribution of this work is permitted, provided the original work is properly cited. 


\section{Zhang et al.}

We propose a new contact structure - a line electrode geometry. This structure effectively improves the CZT detector performance and has a simple readout system. The preliminary results are provided in the paper.

\section{The Line Electrode Geometry}

This new line electrode geometry, shown in Fig. 1, is an efficient method to improve the performance of thick CZT detectors. A line electrode is deposited on one surface of the detector. The planar cathode is on the opposite surface. The two side surfaces that are parallel to the line electrode are also planar cathodes. The three cathodes are connected together with metal contacts. The signal will be read out from the line anode. The electrons migrate toward the line anode and the holes migrate to the cathode. Due to the low electric field and low hole mobility, holes will be strongly trapped, and the signal is mainly induced by the electron movement. This structure is also a single charge sensitive detector.

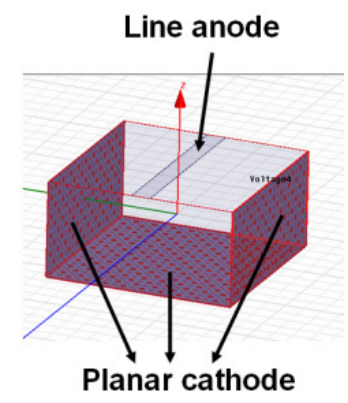

Fig.1. Line electrode geometry scheme.

Compared to coplanar and pixel CZT detectors, this structure is easy to fabricate, and the read out system is very simple. This contact geometry is to some extent similar to high-purity, coaxial germanium detectors, but the CZT crystal is softer and therefore more prone to cleavage. Drilling a hole in CZT can cause severe damage, so it is not an easy operation. The germanium crystal volume is usually large enough for a $10 \mathrm{~mm}$ diameter hole, but for CZT crystals, it is quite difficult to obtain a large volume, up to 50 $\mathrm{mm}$ in diameter, with good uniformity and high quality. With a simple line anode, the operation is simplified and the width of the anode can be adjusted freely to obtain better performance, even for a width of only $1 \mathrm{~mm}$.

\section{Simulation}

In order to analyze the performance of CZT detectors with the line electrode geometry, we simulated the weighting potential, charge collection and spectrum. First, the radiation response was simulated using GEANT4, and then we calculated the electric field and weighting potential using Maxwell's equations. The charges created from incident 
photons will move along the electric field lines: the electrons move toward the line anode and the holes move to the cathode. The induced signal at the electrodes was calculated using the ICC code developed by our research group. The $\mu \tau$ values of holes and electrons assumed for the calculation were $5 \times 10^{-5} \mathrm{~cm}^{2} / \mathrm{V}$ and $7 \times 10^{-3} \mathrm{~cm}^{2} / \mathrm{V}$ respectively.

The spectra shown in Fig. 2 for three sizes of line anode CZT detectors were simulated. Usually the $662 \mathrm{keV}$ photon peak is not obvious in the spectra from 3-mm thick CZT detectors with planar contacts. From our simulations, the best $662 \mathrm{keV}$ energy resolution would be $0.71 \%, 2.87 \%$ and $1.51 \%$ for the $5 \times 5 \times 5 \mathrm{~mm}, 10 \times 10 \times 10 \mathrm{~mm}$ and $20 \times 20 \times 15 \mathrm{~mm}$ line anode CZT detectors, respectively. The resolution varies with the

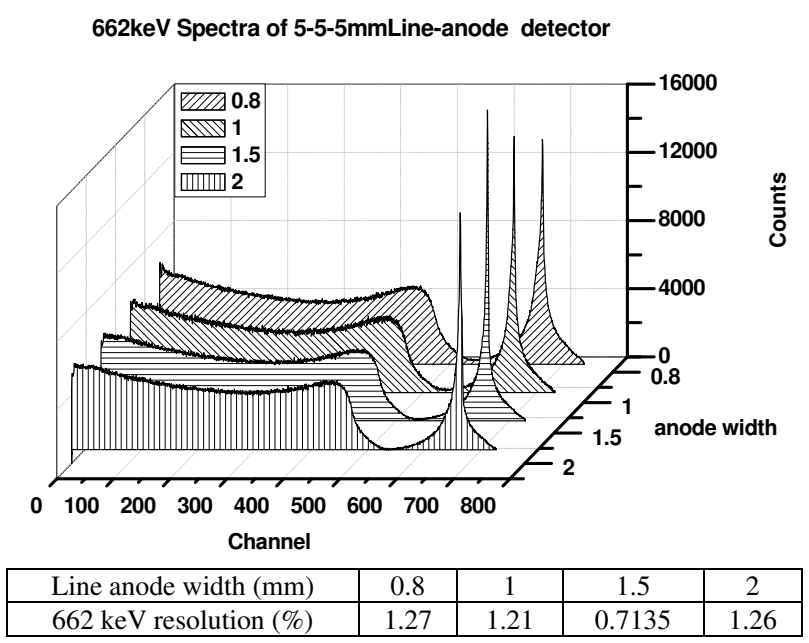

Fig. 2(a). The simulated $662 \mathrm{keV}$ spectra for $5 \times 5 \times 5 \mathrm{~mm}$ CZT detectors with different line anode widths.

662keV Spectra of 10-10-10mmLine-anode detector

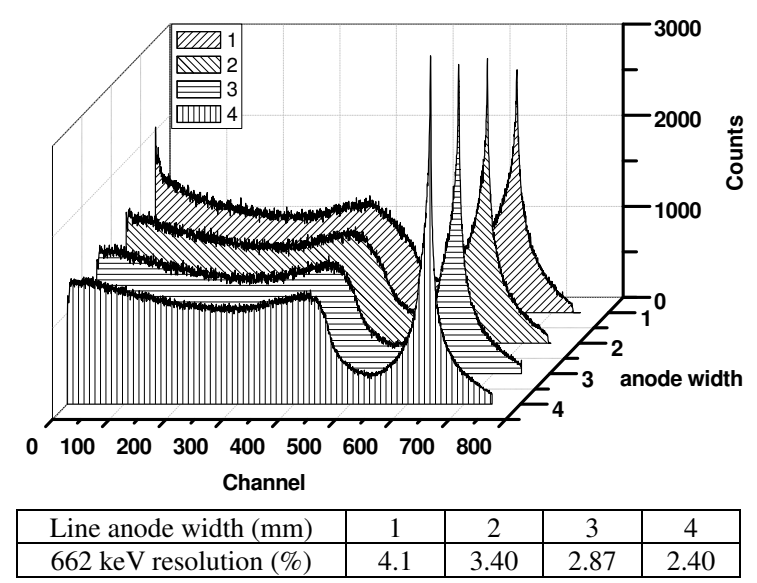

Fig. 2(b). Simulated $662 \mathrm{keV}$ spectra for 10x10x10 mm CZT detectors with different line anode widths. 


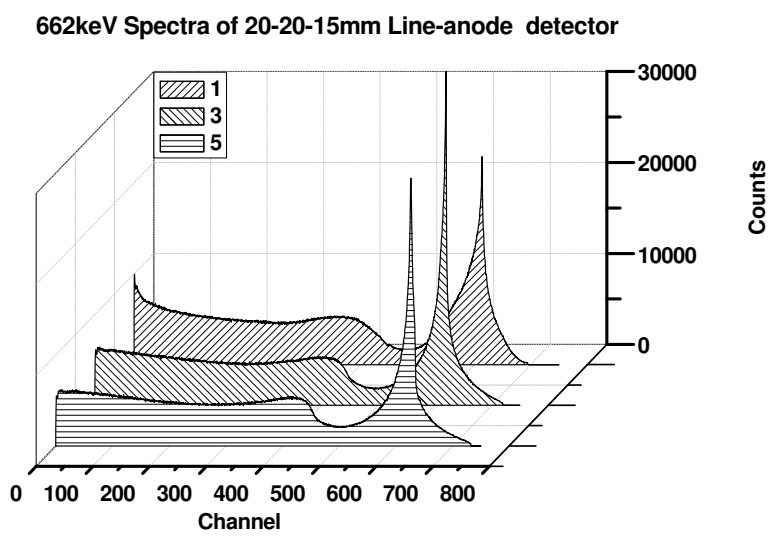

\begin{tabular}{|c|c|c|c|}
\hline Line anode width $(\mathrm{mm})$ & 1 & 3 & 5 \\
\hline $662 \mathrm{keV}$ resolution $(\%)$ & 3.85 & 1.51 & 2.10 \\
\hline
\end{tabular}

Fig. 2(c). Simulated $662 \mathrm{keV}$ spectra for 20x20x15 mm CZT detectors with different line anode widths.

line anode width. We found that the best resolution is obtained through a balance between many detector parameters, such as the area, thickness, line anode width, etc. The narrowest line anode structure did not give the best results, because the electric field is weak and the charge trapping will be strong. The weighting potential of a wider line anode would be spread out and the single charge sensitivity will be affected.

We observed that a high energy tail appeared in all the simulations. To analyze the cause, we calculated the signal amplitude variation with the photon interaction position inside the CZT detector (see Fig. 3). The weighting potential distribution was also calculated. Figure 3 shows that the high energy tail comes from the interaction position close to the area between the side cathode surface and line anode surface. The electric field, the weighting potential and the distance from the readout electrode are suitable for the optimum charge collection at this position.
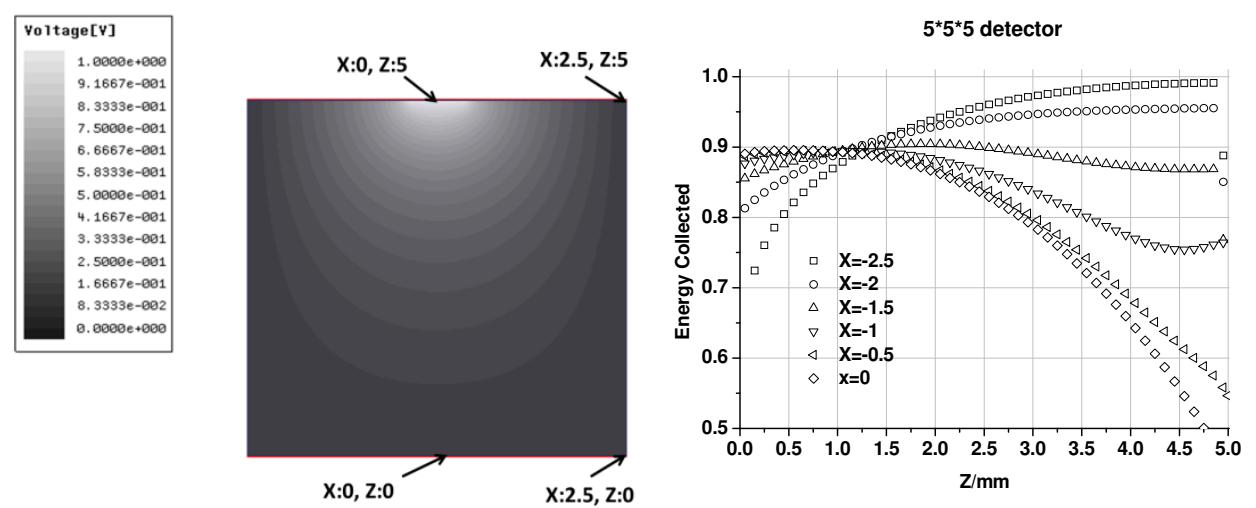

Fig. 3. The relationship between charge collection efficiency and photon interaction position. 
To adjust the signal amplitude to be coincident for good resolution, we decreased the side cathode height. The calculated results are shown in Fig. 4. We can see that the high energy tail has significantly decreased, but it has not disappeared completely. When blocking electrodes were placed between the line anode and the side cathode, the high energy tail has notably diminished, as shown in Fig. 5. The $20 \times 20 \times 15 \mathrm{~mm}$ detector was used for this calculation. The widths of the line anode and the blocking electrodes were $1 \mathrm{~mm}$. The blocking electrodes were placed at both sides of the line anode and powered with a lower voltage than the line anode. The $662 \mathrm{keV}$ energy resolution was improved to $0.99 \%$, but the detection efficiency decreased due to the charge collection by the blocking electrode. To obtain the best energy resolution, the electrode width and location, as well as the voltage difference need to be considered.
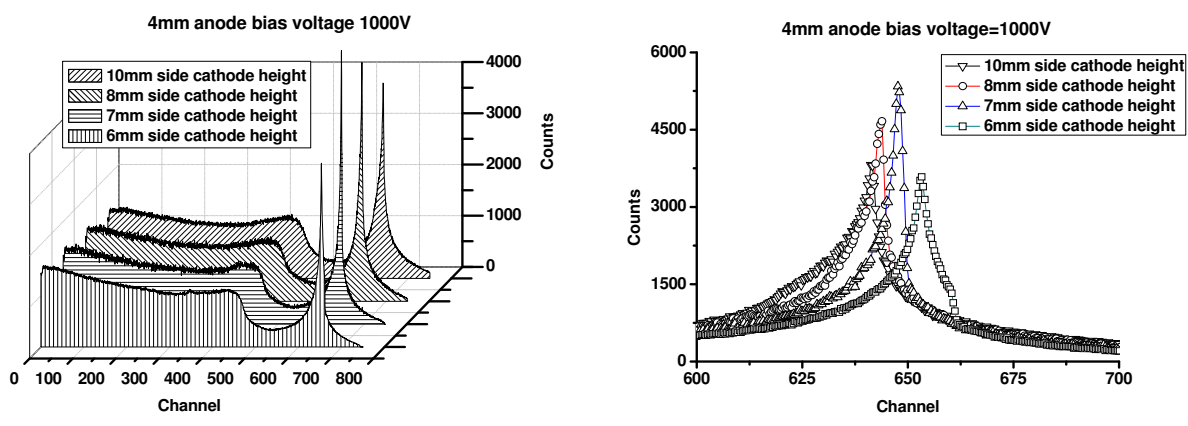

\begin{tabular}{|c|c|c|c|c|c|c|}
\hline Side cathode height $(\mathrm{mm})$ & 5 & 6 & 7 & 8 & 9 & 10 \\
\hline $662 \mathrm{keV}$ resolution $(\%)$ & 2.27 & 1.61 & 0.94 & 1.46 & -- & 2.40 \\
\hline
\end{tabular}

Fig. 4. (Left) The $662 \mathrm{keV}$ spectrum for decreased side cathode height. (Right) Enlarged peak area for comparison.

$662 \mathrm{keV}$ spectra of line anode CZT with blocking electrodes

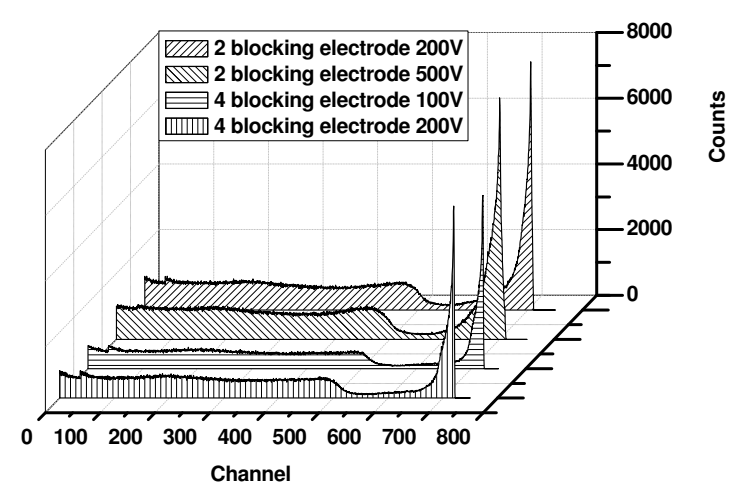

\begin{tabular}{|c|c|c|c|c|}
\hline Number of blocking electrodes & \multicolumn{2}{|c|}{2} & \multicolumn{2}{c|}{4} \\
\hline Voltage difference (V) & 200 & 500 & 100 & 200 \\
\hline $662 \mathrm{keV}$ resolution (\%) & 1.38 & 1.82 & 1.04 & 0.99 \\
\hline
\end{tabular}

Fig. 5. $662 \mathrm{keV}$ spectra of line anode CZT with two and four blocking electrodes. 


\section{Zhang et al.}

Through our research, we found a big difference for lower energy photons incident on the detector from different directions as seen in Fig. 6. The best spectrum is for $60 \mathrm{keV}$ photons incident on the bottom cathode. This is easy to explain: all $60 \mathrm{keV}$ photons were deposited within a $1 \mathrm{~mm}$ thick layer close to the bottom cathode surface. Holes are collected very quickly, and electrons (which all move a similar distance) contribute to the main part of signal. The spectrum for photons incident on the side cathode surface is seriously affected. Although electrons contribute almost to the whole signal, the electron moving distance varies largely, and both the electron trapping and moving distance affect the spectrum.
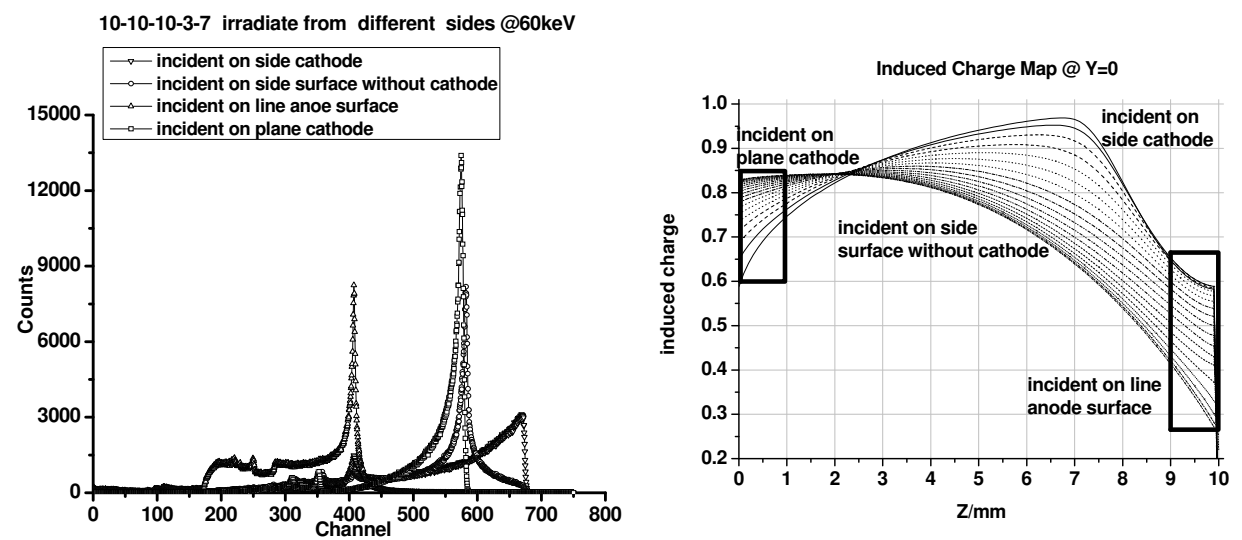

Fig. 6. Spectra of $60 \mathrm{keV}$ photons incident from different directions.

The spectrum for photons incident on the side surface without a cathode shows less peak area. Charges from the interactions spread widely from cathode to anode. It is similar to $662 \mathrm{keV}$ photons penetrating the whole crystal, and the interaction position spreads between the cathode and anode uniformly. The pulse amplitude varies with the interaction position, and the fluctuation decreases the spectrum peak. The spectrum with the peak moving toward the low energy is for photons entering on the line anode surface, and the hole trapping is strong.

Use of blocking electrodes could solve the problem (see Fig. 7). These blocking electrodes collect most of the charge induced by photons incident on both the line anode surface and the side cathode.

\section{Experiments}

In order to verify the simulations and the performance improvement, we fabricated a line electrode geometry CZT detector (see Fig. 8). The size of the detector was $10 \mathrm{x} 10 \mathrm{x}$ $10 \mathrm{~mm}$. The top gold line anode was $4 \mathrm{~mm}$ wide. The height of the side cathode was $7 \mathrm{~mm}$. 


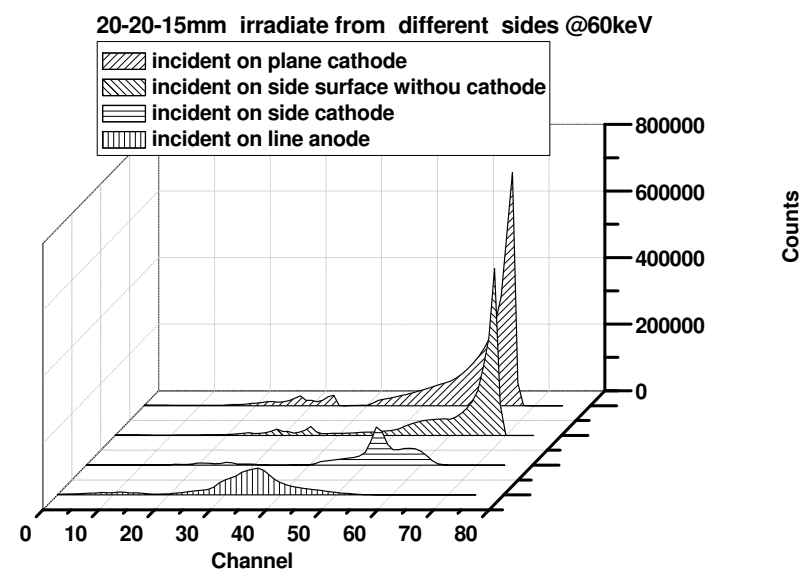

Fig. 7. Spectra of $60 \mathrm{keV}$ photons incident from different directions for blocking electrode structure.

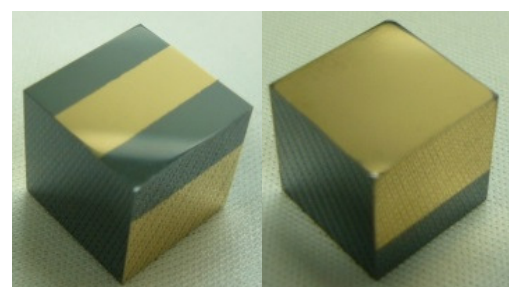

Fig. 8. Line electrode CZT detector of size $10 \times 10 \times 10 \mathrm{~mm}$.

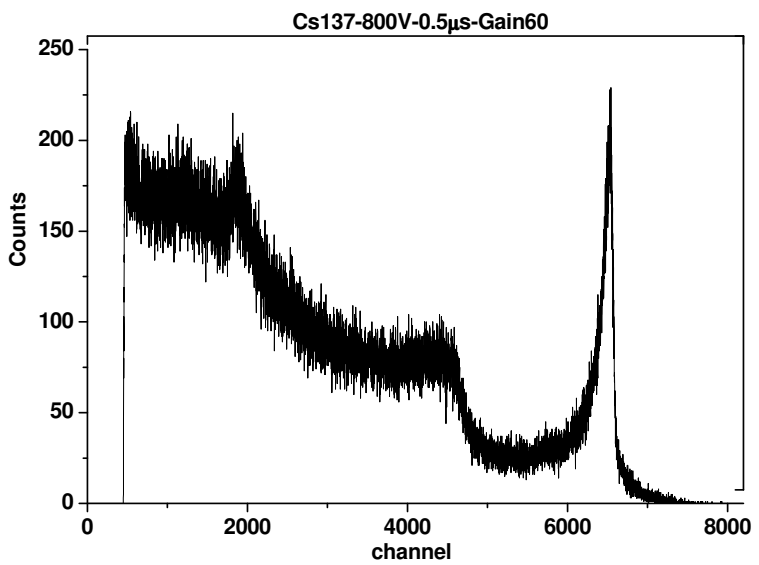

Fig. 9. Measured $662 \mathrm{keV}$ spectrum of the line anode CZT detector.

We irradiated the bottom cathode of the detector with a $662 \mathrm{keV}{ }^{137} \mathrm{Cs}$ source and obtained the spectrum shown in Fig. 9. The energy resolution was found to be $2.17 \%$. Using the test pulse to measure the electronic system noise, the pulse FWHM was calculated to be $1.58 \%$. So the line anode CZT resolution was approximated as $1.49 \%$. It 
was shown that the shaping time and the bias are important parameters for good resolution.

When $60 \mathrm{keV}{ }^{241} \mathrm{Am}$ photons entered the CZT detector from four directions, the spectra shown in Fig. 10 were obtained. We can see that the simulations (Fig. 6) and experiments (Fig. 10) are generally in agreement. Photons incident on the side cathode need to pass $5 \mathrm{~mm}$ thick Teflon, while photons incident on the line anode surface have to go through two PCB board and the distance they have to travel is greater than others. Also, a plane source was used in the simulations while a point source was used for the experiments. This leads to the difference between simulations and experiments. Further experiments are in progress, and the results will be reported in the near future.

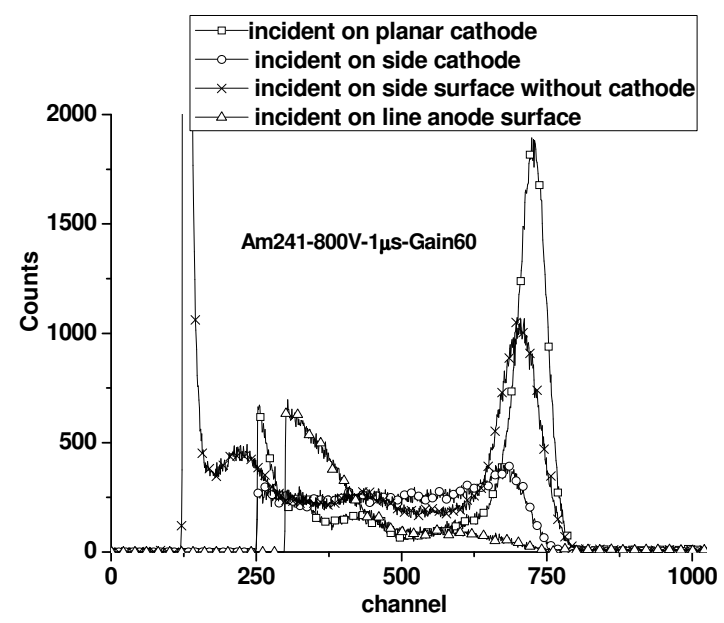

Fig. 10. Measured $60 \mathrm{keV}$ spectra incident from four directions on the line anode CZT detector.

\section{Conclusions}

This paper proposes a new line electrode geometry CZT detector. A line electrode is deposited on one surface of the detector, and the planar cathode is on the opposite surface. The two side surfaces parallel to the line electrode are also planar cathodes. Through simulations and experiments, we determined that the CZT detector performance was obviously improved. Modifying the side cathode height and inserting blocking electrodes further enhanced the energy resolution, especially for low energy photons incident from different directions. Results of the ongoing experiments will be reported in the future.

\section{References}

1. D. S. McGregor, Z. He, H. A. Seifert et al., IEEE Trans. Nucl. Sci. 45(03), 443 (1998).

2. P. N. Luke, Applied Physics Letters 65, 2884 (1994).

3. Csaba Szeles, Derek Bale, Joseph Grosholz et al., Proceedings of SPIE 6319, 631909 (SPIE, Bellingham, WA, 2006), doi:10.1117/12683552.

4. A. Shor, Y. Eisen and I. Mardor, Nucl. Instr. Meth. Phys. Res. A 458, 47 (2001). 